\title{
Effects of sub-chronic branched chain amino acid supplementation on markers of muscle damage and performance variables following 1 week of rigorous weight training
}

Petey Mumford ${ }^{1 *}$, Wesley C Kephart ${ }^{1}$, Anna E McCloskey ${ }^{1}$, Angelia M Holland ${ }^{1}$, Joshua J Shake ${ }^{1}$, C Brooks Mobley ${ }^{1}$, Kaelin C Young ${ }^{2}$, Jordan R Moon ${ }^{3}$, Michael D Roberts ${ }^{1,2}$

From The Twelfth International Society of Sports Nutrition (ISSN) Conference and Expo

Austin, TX, USA. 11-13 June 2015

\section{Background}

We investigated the efficacy of supplementing a branched chain amino acid (BCAA- 3g/d L-Leucine, $1 \mathrm{~g} / \mathrm{d}$ L-Isoleucine and $2 \mathrm{~g} / \mathrm{d}$ L-Valine) supplement compared to a carbohydrate $(\mathrm{CHO})$ control drink in terms of attenuating markers of muscle damage in addition to preserving performance markers following 3 days of intense weight training.

\section{Methods}

Apparently healthy resistance trained males $(\mathrm{n}=30)$ were randomized to either a BCAA group or the $\mathrm{CHO}$ control group. Participants performed preliminary testing (T1) to derive peak quadriceps isometric torque, peak quadriceps isokinetic torque $\left(60^{\circ}\right.$ and $120^{\circ}$ per second), and a $1 \mathrm{RM}$ barbell back squat. The following week, the participants performed $10 \times 5$ repetitions at $80 \%$ of their 1RM barbell back squat for 3 consecutive days. During this experimental intervention antecubital blood was drawn to assess serum myoglobin concentrations, in addition a visual analog scale was utilized in order to measure subjective perceptions of muscular soreness. 48 hours following the third bout of exercise, participants performed post testing (T2) like T1 testing and donated a final blood draw.

\section{Results}

The BCAA group maintained $95 \%$ of their peak isometric torque compared to $86 \%$ for the CHO group ( $\mathrm{p}=0.12$ ). Regarding isokinetic measures at $\mathrm{T} 2$, there was a $92 \%$

${ }^{1}$ School of Kinesiology, Auburn University, Auburn, AL, USA

Full list of author information is available at the end of the article and $88 \%$ maintenance for the BCAA and $\mathrm{CHO}$ group, respectively ( $p=0.39$ ), compared to their respective $\mathrm{T} 1$ values. T2 performance at $120 \%$ s was maintained by $93 \%$ and $96 \%$ of $\mathrm{T} 1$ measurements for the BCAA and $\mathrm{CHO}$ group, respectively $(\mathrm{p}=0.40)$. The BCAA group actually enhanced squat $1 \mathrm{RM}$ by $1 \%$, whereas the $\mathrm{CHO}$ group experienced a 3\% decrement; however, this difference failed to reach significance $(\mathrm{p}=0.92)$. Serum myoglobin concentrations increased as a function of time, and there was no difference between groups $(\mathrm{p}=0.31)$. Lastly, perceptions of muscular soreness were also not differentially altered between groups $(\mathrm{p}=0.09)$.

\section{Conclusions}

In conclusion, while a BCAA supplement did not appear to enhance recovery benefits compared to a $\mathrm{CHO}$ control, a few areas of performance were bolstered to a point of practical importance regarding high level competition.

\section{Authors' details}

'School of Kinesiology, Auburn University, Auburn, AL, USA. ${ }^{2}$ Edward Via College of Osteopathic Medicine - Auburn Campus, Auburn, AL, USA. ${ }^{3}$ MusclePharm Sports Science Institute, Denver, CO, USA.

Published: 21 September 2015
doi:10.1186/1550-2783-12-S1-P29
Cite this article as: Mumford et al: Effects of sub-chronic branched

chain amino acid supplementation on markers of muscle damage and performance variables following 1 week of rigorous weight training. Journal of the International Society of Sports Nutrition 2015 12(Suppl 1):P29. 